\title{
Complete trisomy of chromosome 9: karyotyping and morphological alterations in newborn
}

\author{
Carolina Leão de Moraes*, Nádia Aparecida Bérgamo², Natália Cruz e Melo³, \\ Roberta Machado de Oliveira Frota Curado ${ }^{4}$, Waldemar Naves do Amaral ${ }^{5}$
'Universidade Federal de Goiás (UFG), Instituto de Ciências Biológicas, Faculdade de Medicina, Departamento de Ginecologia e Obstetrícia, Goiânia, GO, Brasil
${ }^{2}$ Universidade Federal de Goiás (UFG), Instituto de Ciências Biológicas, Goiânia, GO, Brasil
${ }^{3}$ Universidade Federal de São Paulo (UNIFESP), Departamento de Ginecologia, Laboratório de Ginecologia Molecular, São Paulo, SP, Brasil ${ }^{4}$ Universidade Federal de Goiás (UFG), Instituto de Ciências Biológicas, Goiânia, GO, Brasil \\ ${ }^{5}$ Universidade Federal de Goiás (UFG), Faculdade de Medicina, Departamento de Ginecologia e Obstetrícia, Goiânia, GO, Brasil
}

\begin{abstract}
Objectives: To Describe the complete trisomy of chromosome 9 and morphological changes of the newborn. Methods: This case report described $47 X X+9$ in newborn of 19 year-old healthy primigesta, in Goiania, Brazil. Ultrasound was used in the 20th week of gestation to analyze multiple fetal malformations. Cytogenetic analysis (Bandage $\mathrm{G}$ ) was used to identify the karyotype of the newborn and parents. Results: The cytogenetic analysis (G banding), showed that the fetus was female and had a 47,XX,+9 karyotype. The delivery was carried out by cesarean section at the 34th week. The evolution was unfavorable, baby survived for approximately 65 minutes and showed neonatal ocular hypertelorism, micrognathia, low ear implantation with pavilion auricle anomaly, ambiguous genitalia, spina bifida, and clubfoot. The analysis performed by chromosomal banding technique with umbilical cord lymphocytes culture showed of $47 X X+9$ karyotype in all 20 surveyed cells. Karyotyping of peripheral blood parents was realized but both not showed chromosomal changes. Conclusions: According to our knowledge, this is the second case of $47 X X+9$ in Brazil and the detection of morphological changes caused by rare syndromes during pregnancy may help in diagnosis and appropriate clinical management because they direct families to genetic tests that can essential for the future of gestation.
\end{abstract}

Keywords: trisomy 9; congenital malformations; fetal ultrasound; general cytogenetics; prenatal cytogenetics and genetic counselling.

\section{Introduction}

Trisomy 9 is a rare chromosomal abnormality usually associated with first-trimester miscarriage. Complete trisomy 9 is a lethal diagnosis, and most fetuses diagnosed die prenatally or during the early postnatal period. Most often, it is seen in mosaic form. ${ }^{1-3}$

Mosaic and non-mosaic patients usually exhibit similar clinical features. However, both forms of trisomy 9 differ concerning survival. In fact, patients with mosaicism survive longer than non-mosaics. The non-mosaic form is rarely seen at near term. When they come to birth, the babies usually die in the neonatal period, and only $25 \%$ live more than one week. ${ }^{2}$

The occurrence of complete 9 trisomy has been estimated at 1 per 1000 recognized conceptions. However, the most of pregnancies with trisomy 9 results in miscarriages in the first trimester of pregnancy, making the diagnostic of this chromosomal abnormality relatively uncommon. Additionally, the cytogenetic is not performed in most abortions. ${ }^{2,3}$

The features of trisomy 9 are mental and growth retardation, facial dismorphism (small ears, microphthalmia, bulbous nose, small mouth, high palate), central nervous system abnormalities (hydrocephalus, malformation, Dandy-Walker

Financial support: None.

Conflicts of interest: The authors declare no conflicts of interest.

Submitted: October 03, 2018

Accepted: October 16, 2019.

The study was carried out at Universidade Federal de Goiás (UFG), Goiânia, GO, Brasil.

Copyright Moraes et al. This is an Open Access article distributed under the terms of the Creative Commons Attribution License, which permits unrestricted use, distribution, and reproduction in any medium, provided the original work is properly cited. 
syndrome, holoprosencephaly), congenital heart disease (most commonly ventricular septal defects), genitourinary anomalies (genital hypoplasia, cryptorchidism, cystic kidneys, hydronephrosis), and skeletal anomalies (dislocations, deformities). ${ }^{1,3,4}$

The diagnosis of trisomy 9 is performed through the identification of chromosomal abnormalities in cells of patient suspected prior diagnosis of trisomy using cytogenetic or molecular techniques, such as cytogenetic analysis by $\mathrm{G}$ banding and fluorescence in situ hybridization (FISH). ${ }^{2}$ In addition, published data show that ultrasound examination in the second quarter of prenatal care can show morphological changes in the baby that assist in the diagnosis of trisomy 9 and clinical management. The diagnosis of trisomy 9 exclusively by prenatal ultrasound is difficult since the sonographic findings are nonspecific, due to overlap with other trisomies, such as 18 and 13.2,3 In context, the objective of the present study was to describe the phenotypic characteristics of a newborn with complete trisomy of chromosome 9.

\section{Methods}

The pregnant woman suspected of have fetus with congenital anomaly was attended in the fetal medicine department of the Department of Gynecology and Obstetrics of the Clinical Hospital of the Federal University of Goiás (HCIUFG) in 2015. The Ethics Committee (HCIUFG) approved the study protocol. The term of Informed Consent was signed by the parents of the newborn authorizing the conduct of the research. The parents were submitted to a questionnaire on gynecological and obstetric history and consumption of teratogenic substances.

\section{Analysis of chromosomal alterations}

Were collected peripheral blood samples from parents, and amniocentesis and cordocentesis fetal for classical cytogenetic analysis. The karyotype was performed after culture of lymphocytes. The cells were stimulated with phytohemagglutinin, to induce cell division and obtain cells in metaphase for chromosome analysis. In aseptic conditions 0.5 to $1.0 \mathrm{ml}$ of whole blood was incubated in RPMI culture medium and fetal bovine serum at $37^{\circ} \mathrm{C}$. After 72 hours of incubation, colchicine was added to disrupt the division of cells into metaphase, following the protocol described by Moorhead et al. ${ }^{5}$ with modifications. After the processing of the culture the GTG banding was made, following the protocol of Scheres, ${ }^{6}$ with modifications. An average of 20 metaphase cells were analyzed for determination of the patient's karyotype.

\section{Analysis of phenotypic feature}

Phenotypic alterations were observed through ultrasonography at the 22nd week of gestation. After birth, the circumference of the head, thorax and abdomen, and weight were measured. In addition, the morphological abnormalities presented by the newborn were analyzed.

\section{Case description}

A pregnant woman, KPS white, 19-year-old, primigravida was referred to fetal medicine service due to multiple fetal malformations viewed on ultrasound performed at the 20th week of gestation: agenesis of the cerebellar vermis, nuchal fold change, cranial deformity, cardiomegaly, multicystic right kidney, left kidney with pyelectasis, and severe oligohydramnios (Figure 1). Her husband was 23 years old. The pregnant woman and her husband were healthy and not consanguineous. The mother related that had first cousins with mental problems, though the describe neurological diseases were not specified. The parents refused to have a habit of smoking, drinking alcohol, make the using drugs or prescription drugs. In addition, during pregnancy, there was no infectious process or other health problem.

Suspicion of any genetic alteration was confirmed by chromosomal analysis of amniotic fluid cells harvested in the 21 th week of pregnancy. The amniocytes were grown, and, after cytogenetic analysis (G banding), it could be seen that the fetus was female and the karyotype $47, X X,+9$ was not mosaic (Figure 1). The patient remained under monitoring of the institution. The delivery was carried out by caesarean section at the 34th week. The newborn female presented as 1565 grams, $40 \mathrm{~cm}$ in length, head circumference of $31 \mathrm{~cm}$, chest $25 \mathrm{~cm}$ and waist circumference of $24 \mathrm{~cm}$. The evaluation confirmed neonatal ocular hypertelorism, micrognathia, low ear implantation with pavillion auricle anomaly, ambiguous genitalia, spine bifida, and clubfoot (Figure 2). The baby was resuscitated for approximately 65 minutes then subsequently died.

For confirmation of chromosomal disorder, the chromosomes of the umbilical cord lymphocytes were cultured and analyzed by banding technique. Results showed a nonmosaic constitution of trisomy $9(47, X X,+9)$ in all 20 surveyed cells, confirming the cytogenetic analysis of amniotic fluid. Who asked karyotyping of peripheral blood progenitors, but both had karyotypes with no chromosomal changes. 


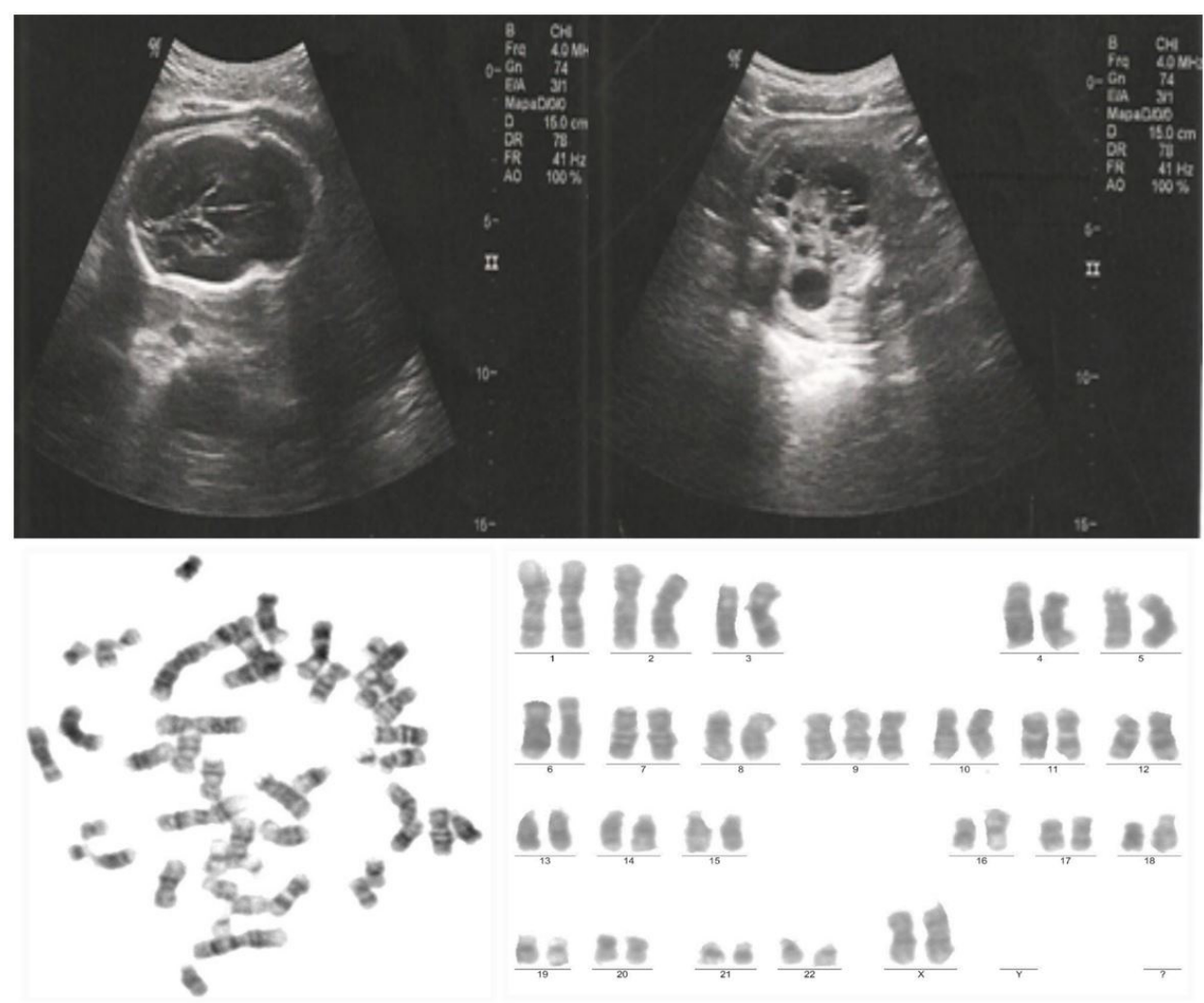

Figure 1. Ultrasound examination and karyotype of fetus with complete trisomy 9. Above right and left; multiple fetal malformations viewed on ultrasound performed at the 20th week of gestation: agenesis of the cerebellar vermis, nuchal fold change, cranial deformity, cardiomegaly, multicystic right kidney, left kidney with pyelectasis, and severe oligohydramnios. Below right and left; The amniocytes were grown, and after cytogenetic analysis (G banding), it could be seen that the fetus was female and the karyotype $47 X X+9$ was not mosaic.

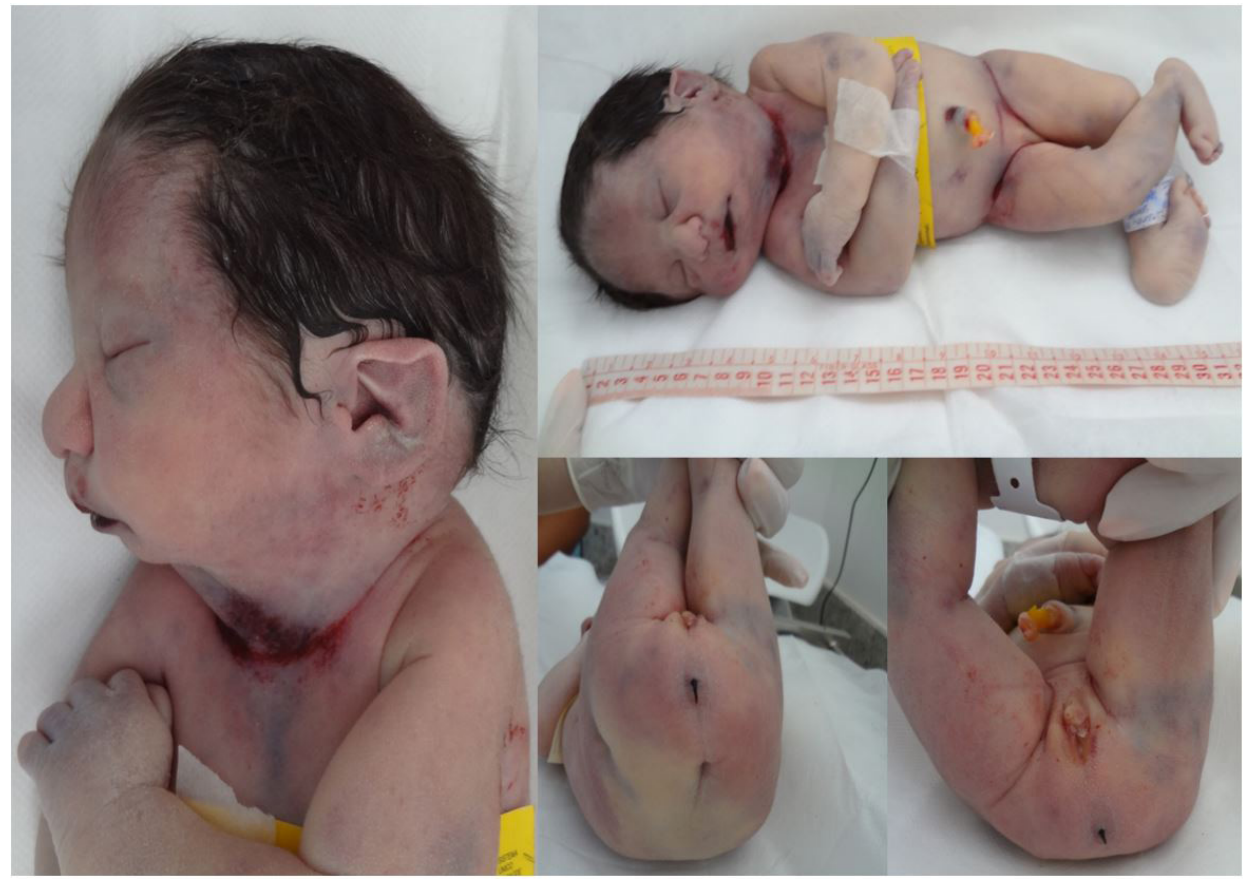

Figure 2. Morphological alterations of the newborn with complete trisomy 9. Above right. The newborn female presented as 1565 grams, $40 \mathrm{~cm}$ in length and and clubfoot. Left. neonatal ocular hypertelorism, micrognathia, low ear implantation with pavillion auricle anomaly. Below right and left. ambiguous genitalia and spine bifida. 


\section{Discussion}

The first case of complete trisomy of chromosome 9 was reported by Feingold and Atkins. ${ }^{1}$ It was a male child with multiple malformations, a product of 40 weeks of gestation. In the culture of lymphocytes, karyotype $47 X Y+9$ was observed in 63 cells examined. ${ }^{1}$ The prominent clinical features observed in this case were small palpebral fissures, bulbous nose, low-set ears, genital hypoplasia, and malformations of the heart, brain, and skeleton. The fetus survived for 28 days, being the longest survival period mentioned in the literature of a child with complete trisomy. ${ }^{2,7}$

The study of Katayama et al. ${ }^{8}$ reported a newborn with complete trisomy 9 with the head higher than normal and clover shaped. In addition to these features, there were low ear implantation, malformed ears, micrognathia, bulbous nose, brain abnormalities, congenital heart disease, abnormal hands and feet, cryptorchidism, micropenis, and premature death.

Seabright et al. ${ }^{7}$ described another child with complete trisomy 9 with a similar phenotype who lived for 16 hours. Subsequently, Mantagos et al. ${ }^{9}$ described 2 cases of complete trisomy 9 in live births and found a striking phenotypic similarity to the previously reported cases.

In Brazil, Murta and Vargas ${ }^{4}$ reported a fetus with complete trisomy 9 in a primiparous mother 30 years of age. In the 14th week, the fetal death occurred. Pathological examination after death showed a male fetus with multiple anomalies, such as ocular hypertelorism, microtia and low implantation, left unilateral cleft lip, high palatal bow (in gothic arch) with incomplete cleft, large septal, absence of attachment of the small and large intestines, and mild ectasia of the pelvis and renal calyces bilaterally. Unlike this study, our case report showed a newborn female in a younger pregnant woman, 19 years old. However, we noted similarities, for both pregnant women were experiencing their first pregnancy, and the fetuses had malformations in common.

Parents of the newborn in our study were healthy. The same characteristic was quoted by Henedi et al., ${ }^{2}$ who described complete trisomy 9 in a newborn weighing 1250 gram. The patient had unusual features, such as anal atresia, cysts in the ventricles, and hemispheric fissures. Similar to our study, the external genitalia were ambiguous, and sex determination was carried out only after karyotype results.

Ultrasound examination of a16 week-old fetus with complete trisomy 9, presented by the case report of Pruksanusak et al. ${ }^{3}$ showed a fetus with a strawberry shaped head, moderately hyperechoic intestine, overlapping fingers, and a single umbilical artery. The fetal echocardiogram showed situs solitus and abnormal deviation from the left axis. Furthermore, abnormal cardiac calcification in both the ventricular walls and interventricular septum was also identified. Cordocentesis was performed, and the karyotype confirmed complete trisomy 9 in the female fetus. In addition, genetic counseling was provided for the family, and termination of the pregnancy was performed at 21 weeks and 5 days. In our case report, genetic counseling was also provided, however, the mother chose not to terminate the pregnancy. Contrasting other trisomies, such as trisomy 21, complete trisomy 9 does not seem to correlate with maternal age, but it is more common in women younger than 35 years, ${ }^{10}$ and our findings confirm this correlation. However, Pruksanusak et al. ${ }^{3}$ reported a maternal age of 37 years, this would suggest that it does correlate with maternal age.

In most reported cases of complete trisomy 9 is not determined the origin of the extra chromosome. However, Seabright et al. ${ }^{7}$ analyzed the variants Band $\mathrm{C}$ of the three chromosomes 9 in patient of trisomy and indicated that two chromosomes of patient they were possibly of maternal origin.

The risk of chromosomal abnormality in the second pregnancy of a woman who had a fetus with trisomy 9 , whether alive or unborn, is unknown because of the small number of reported cases. ${ }^{9}$

Cytogenetic findings should be interpreted considering the clinical situation. Stipoljev et al., ${ }^{11}$ in 1975 performed the first prenatal diagnosis of complete trisomy 9 by amniocentesis. Thus, has been suggested that the collection of periumbilical blood can be used to clarify the results of mosaicism in samples from amniocentesis or chorionic villi. ${ }^{12}$

\section{Conclusion}

Complete trisomy 9 is a genetic abnormality is rare worldwide. According to our knowledge, this is the second case of complete trisomy 9 in Brazil. ${ }^{4}$ Reports about this genetic abnormality are extremely important, as they present morphological changes of the fetus or newborn that assist in the diagnosis and appropriate clinical management. In Brazil, there are legislative policies to allow termination of pregnancies in cases of confirmed congenital abnormalities thus, the elucidation of early diagnosis in the prenatal period allows the family to decide on the course of the pregnancy. To establish an appropriate diagnosis of complete trisomy 9, tracking defects by ultrasound associated with prenatal cytogenetic study (amniocentesis or fetal blood sampling) becomes essential. In this scenario, genetic counseling for parents is important for the family's decision about the future of the current gestation and the next generations. 


\section{Acknowledgements}

We appreciate the financial support from Coordenação de Aperfeiçoamento de Pessoal de Nível Superior (CAPES) for providing PhD scholarship for Moraes CL, NC Melo and RMOF Curado.

\section{References}

1. Feingold M, Atkins L. A case of trisomy 9. J Med Genet. 1973;10(2):184-7. http://dx.doi.org/10.1136/jmg.10.2.184. PMid:4714588.

2. Henedi MMA, Mohammed FM, Masoud HA, Abualhasan SJ, Al Awadiet SA. Trisomy 9 syndrome in a neonate with unusual features. Egypt J Med Hum Genet. 2009;10(2):238-42.

3. Pruksanusak N, Rujirabanjerd S, Kanjanapradit K, Kor-anantakul O, Suntharasaj T, Suwanrath C, et al. Prenatal diagnosis of complete trisomy 9 with a novel sonographic finding of heart calcification. J Ultrasound Med. 2014;33(10):1871-3. http://dx.doi. org/10.7863/ultra.33.10.1871. PMid:25253836.

4. Murta CGV, Vargas PRM. Trissomia do cromossomo 9 associada com o aumento da transluscência nucal: correlação ultrassonografica e anatomopatológica. Radiol Bras. 2001;34(2):105-8. http://dx.doi.org/10.1590/S0100-39842001000200010.

5. Moorhead PS, Nowell PC, Mellman WJ, Battips DM, Hungerford DA. Chromosome preparations of leukocites cultured from human peripheral blood. Exp Cell Res. 1960;20(3):613-6. http://dx.doi.org/10.1016/0014-4827(60)90138-5. PMid:13772379.

6. Scheres VMJC. Identification of two Robertsonian translocations with a Giemsa banding technique. Hum Genet. 1972;15(3):253-6. http://dx.doi.org/10.1007/BF00702361. PMid:4117149.

7. Seabright M, Gregson N, Mould S. Trisomy 9 associated with enlarged 9qh segment in a liveborn. Hum Genet. 1976;34(3):323-5. http://dx.doi.org/10.1007/BF00295299. PMid:1002157.

8. Katayama KP, Wilkinson EJ, Herrmann J, Glaspey JC, Agarwal AB, Roesler MR, et al. Clinical delineation of trisomy 9 syndrome. Obstet Gynecol. 1980;56(5):665-8. PMid:7432742.

9. Mantagos S, McReynolds JW, Seashore MR, Breg WR. Complete trisomy 9 in two liveborn infants. J Med Genet. 1981;18(5):37782. http://dx.doi.org/10.1136/jmg.18.5.377. PMid:7328618.

10. Stipoljev F, Kos M, Kos M, Miskovi B, Matijevic R, Hafner T, et al. Antenatal detection of mosaic trisomy 9 by ultrasound: a case report and literature review. J Matern Fetal Neonatal Med. 2003;14(1):65-9. http://dx.doi.org/10.1080/jmf.14.1.65.69. PMid:14563095.

11. Francke U, Benirschke K, Jones OW. Prenatal diagnosis of trisomy 9. Humangenetik. 1975;29(3):243-50. PMid:1165103.

12. Sherer DM, Wang N, Thompson HO, Peterson JC, Miller ME, Metlay LA, et al. An infant with trisomy 9 mosaicism presenting as a complete trisomy 9 by amniocentesis. Prenat Diagn. 1992;12(1):31-7. http://dx.doi.org/10.1002/pd.1970120105. PMid:1557309.

\section{*Correspondence}

Carolina Leão de Moraes

Universidade Federal de Goiás (UFG), Instituto de Ciências Biológicas, Faculdade de Medicina, Departamento de Ginecologia e Obstetrícia

Rua 235, s/n, Setor Leste Universitário

CEP 74605-050, Goiânia, GO, Brasil

Tel.: +55 (62) 3209-6151

E-mail: carolina.leao.moraes@gmail.com

\section{Authors information}

CLM - Msc. (Ginecologia e Obstetrícia), NAB - PhD. (Citogenética), NCM - Msc. (Biologia Molecular), RMOFC - Ms. (Citogenética), WNA - PhD (Ginecologia e Obstetrícia).

\section{Authors contribution}

CLM and WNA conceived and designed the experiments. NAB and RMOFC performed the experiments. CLM, NCM, WNA, NAB and RMOFC wrote the paper. 